\title{
Hospitalização por Infarto Agudo do Miocárdio: Um Registro de Base Populacional
}

\author{
Hospitalization for Acute Myocardial Infarction: A Population-Based Registry
}

Leonardo Alves ${ }^{1,2,3(0)}$ e Carisi Anne Polanczyk ${ }^{1,4}$

Universidade Federal do Rio Grande do Sul, ${ }^{1}$ Porto Alegre, RS - Brasil

Universidade Federal do Rio Grande, ${ }^{2}$ Rio Grande, $R S$ - Brasil

Hospital Santa Casa do Rio Grande - Hospital de Cardiologia, ${ }^{3}$ Rio Grande, RS - Brasil

Hospital de Clínicas de Porto Alegre, ${ }^{4}$ Porto Alegre, $R S$ - Brasil

\section{Resumo}

Fundamento: O infarto agudo do miocárdio com supradesnivelamento do segmento ST (STEMI) é uma das principais apresentações clínicas da cardiopatia isquêmica. Dados de base populacional são relevantes para entendimento contemporâneo da epidemiologia da doença.

Objetivo: Descrever incidência, manejo terapêutico, desfechos clínicos hospitalares e eventos cardiovasculares do primeiro ano de seguimento dos indivíduos hospitalizados por STEMI.

Métodos: Estudo de coorte prospectiva de base populacional com registro consecutivo das hospitalizações por STEMI em uma cidade do Sul do Brasil entre 2011 e 2014. Foram incluídos indivíduos com STEMI que apresentaram sintomas de isquemia miocárdica aguda nas últimas 72 horas. Os valores de $p<0,05$ foram considerados significativos.

Resultados: A incidência anual de hospitalizações por STEMI foi de 108 casos por 100.000 habitantes. A incidência ajustada foi maior entre os mais velhos (risco relativo 64,9; IC95\% 26,9 - 156,9; p para tendência linear < 0,001) e entre os homens (risco relativo 2,8; IC95\% 2,3 - 3,3; $p<0,001$ ). Ocorreram 530 hospitalizações durante o período avaliado e a taxa de reperfusão foi de $80,9 \%$. A mortalidade hospitalar e a taxa de eventos cardiovasculares em 1 ano foram, respectivamente, $8,9 \%$ e $6,1 \%$. Os mais velhos apresentaram maior mortalidade hospitalar (risco relativo 3,72; IC95\% 1,57 - 8,82; $p$ para tendência linear = 0,002) e mais eventos cardiovasculares em 1 ano (hazard ratio 2,35; IC95\% 1,12-4,95; $p=0,03)$.

Conclusão: Este registro demonstra abordagem terapêutica e mortalidade hospitalar semelhante às observadas em países desenvolvidos. Entretanto, a taxa de hospitalizações foi maior comparada com esses países. (Arq Bras Cardiol. 2020; 115(5):916-924)

Palavras-chave: Infarto do Miocárdio/mortalidade; Hospitalização; Epidemiologia; Fatores de Risco; Prevenção e Controle; I ntervenção Coronária Percutânea.

\begin{abstract}
Background: ST-segment elevation myocardial infarction (STEMI) is one of the main clinical manifestations of ischemic heart disease. Populationbased data are relevant to better understand the current epidemiology of this condition.

Objective: To describe the incidence, therapeutic management, hospital clinical outcomes and cardiovascular events in the first year of followup of individuals hospitalized for STEMI.

Methods: Population-based prospective cohort study with consecutive registries of hospitalization for STEMI in a city in southern Brazil from 2011 to 2014. It included patients with STEMI who presented acute myocardial ischemia symptoms in the last 72 hours. A p-value < 0.05 was considered significant.
\end{abstract}

Results: The annual incidence of STEMI hospitalizations was 108 cases per 100,000 inhabitants. Adjusted incidence was higher among older individuals (relative risk 64.9; 95\% Cl 26.9-156.9; $p$ for linear trend < 0.001) and among men (relative risk 2.8; 95\% Cl 2.3-3.3; $p<0.001$ ). There were 530 hospitalizations in the period under evaluation and the reperfusion rate reached $80.9 \%$. Hospital mortality and the one-year follow-up cardiovascular event rate were, respectively, $8.9 \%$ and $6.1 \%$. The oldest patients had higher hospital mortality (relative risk 3.72; 95\% $\mathrm{Cl} 1.57-8.82 ; \mathrm{p}$ for linear trend $=0.002$ ) and more one-year follow-up cardiovascular events (hazard ratio 2.35; 95\% Cl 1.12-4.95; $p=0.03$ ).

Correspondência: Leonardo Alves •

Universidade Federal do Rio Grande - Departamento de Medicina - Gen Osório. CEP 96201-900, Rio Grande, RS - Brasil

E-mail: leoalvesrg@gmail.com

Artigo recebido em 23/08/2019, revisado em 01/12/2019, aceito em 27/12/2019

DOI: https://doi.org/10.36660/abc.20190573 
Conclusion: This study shows that both the therapeutic approach and hospital mortality are similar to the ones found in developed countries. However, the hospitalization rate was higher in these countries.(Arq Bras Cardiol. 2020; 115(5):916-924)

Keywords: Myocardial/mortality; Hospitalization; Epidemiology; Risk Factors; Prevention and Control; Percutaneous Coronary Intervention.

Full texts in English - http://www.arquivosonline.com.br

\section{Introdução}

As doenças cardiovasculares (DCV) são a principal causa de mortalidade em adultos de ambos os sexos em todo o mundo, assim como a causa dominante das mortes prematuras; destas, cerca de $75 \%$ ocorrem em países de baixa e média renda. ${ }^{1}$ No Brasil, as DCV, mesmo com tendência para o declínio, também são a principal causa de óbitos em adultos. ${ }^{2}$

A cardiopatia isquêmica é responsável pela maioria das mortes devido às DCV. A Organização Mundial da Saúde (OMS) estima que 17,7 milhões de pessoas morreram por DCV em 2015; destas, 7,4 milhões foram devido à cardiopatia isquêmica que, no Brasil, também é a causa dominante entre as DCV. ${ }^{2}$

O infarto agudo do miocárdio com supradesnivelamento do segmento ST (STEMI) é uma das principais apresentações clínicas da cardiopatia isquêmica, e seu reconhecimento clínico é de fundamental importância para imediata estratégia terapêutica. Estudos mostram que, apesar da diminuição da incidência, a mortalidade por STEMI permanece sem relevante variação. . $^{3,4}$

No Brasil, não há dados de base populacional sobre a taxa de hospitalização por STEMI. Por sua vez, as informações sobre hospitalizações por STEMI, tais como mortalidade e taxa de reperfusão, são provenientes, em sua maioria, de registros que apresentam limitações. Esses registros ficam restritos a um hospital ou, se multicêntricos, não são representativos da população, pois decorrem da amostragem por conveniência (seleção por convite ou voluntária), o que pode enviesar as estimativas. Outras limitações são o recrutamento não consecutivo dos pacientes e critérios restritos de elegibilidade, como a seleção de pacientes com sintomas com duração de até 12 horas, sabendo-se que a apresentação após esse período está associada à maior mortalidade.

Nesse contexto, o objetivo deste estudo é descrever a incidência, a abordagem terapêutica, os desfechos clínicos hospitalares e os eventos cardiovasculares do primeiro ano de seguimento de indivíduos hospitalizados por STEMI em uma região circunscrita do Sul do Brasil. Avaliar essas informações é relevante pela elevada incidência dessa doença e escassez de estudos de base populacional ${ }^{5}$ no Brasil. Além disso, registros são uma maneira efetiva de abordar a implementação das diretrizes clínicas e fontes de dados para gestores, profissionais e pesquisadores da área da saúde. ${ }^{6,7}$

\section{Métodos}

\section{Desenho do estudo}

Foi conduzido um estudo de coorte prospectiva com registro consecutivo das hospitalizações por STEMI na cidade do Rio Grande (RS) entre janeiro de 2011 e dezembro de 2014. Essa cidade está localizada no Sul do Brasil e compreende cerca de 200 mil habitantes, cuja maioria reside na zona urbana (Censo Demográfico 2010). No Rio Grande, há uma emergência aberta - Hospital de Cardiologia/Santa Casa do Rio Grande - que é o centro de referência no município para tratamento da síndrome coronariana aguda e, consequentemente, para onde todos indivíduos com sintomas sugestivos dessa condição se dirigem ou são encaminhados, tornando o nível de perda de encaminhamento muito baixo. Na cidade, não há linha de cuidado do infarto agudo do miocárdio, de modo que o paciente procura espontaneamente os estabelecimentos de saúde.

\section{Critérios de elegibilidade}

Para serem incluídos no estudo, os indivíduos deveriam preencher os seguintes critérios à admissão hospitalar: (1) idade $\geq 18$ anos e serem moradores da cidade do Rio Grande; (2) apresentarem sintomas de isquemia miocárdica aguda nas últimas 72 horas; (3) apresentarem eletrocardiograma na admissão com supradesnivelamento do segmento ST (SST) com $\geq 1 \mathrm{~mm}$ em duas ou mais derivações contíguas periféricas ( $\geq 2 \mathrm{~mm}$ em derivações precordiais) ou novo ou presumivelmente novo bloqueio de ramo esquerdo; e (4) elevação dos marcadores de necrose miocárdica (troponina ou CK-MB).

Os pacientes que não tiveram marcadores de necrose miocárdica mensurados foram incluídos desde que apresentassem sintomas típicos de isquemia miocárdica aguda associados a SST inequívoco que justificassem imediata terapia de reperfusão. Os pacientes que apresentaram novos STEMI durante o período do estudo foram incluídos como um novo evento desde que ocorresse 28 dias após o primeiro evento.

Foram excluídos os pacientes com SST transitório, que, neste estudo, foi definido como resolução espontânea associada à melhora da dor antes do início da terapia de reperfusão. Os reinfartos, ${ }^{8}$ definidos como novos episódios até 28 dias do evento incidente, também foram excluídos, contribuindo apenas para o seguimento clínico.

\section{Cálculo amostral}

Com o objetivo de calcular o tamanho de amostra para a taxa de hospitalizações, foram utilizados os seguintes parâmetros: taxa esperada de 100 casos/100.000 habitantes/ ano, ${ }^{9}$ precisão de 20 casos/100.000 habitantes/ano e nível de confiança de $95 \%$. Esse processo resultou em um total de 95.941 indivíduos - a população-alvo deste estudo é de cerca de 160.000 habitantes (Censo Demográfico, 2010). Para o cálculo do tamanho de amostra para mortalidade hospitalar, foram empregados os seguintes parâmetros: proporção esperada de $10 \% ;^{9}$ precisão de 2,5 pontos percentuais; 
e nível de confiança de 95\%. Esse processo totalizou 554 pacientes. Com base na taxa de hospitalizações esperada de 100/100.000/ano e na população-alvo de 160.000 indivíduos, seriam necessários 4 anos para alcançar a amostra calculada.

\section{Coleta de dados}

\section{As informações coletadas foram:}

a) Sociodemográficas - idade, sexo, atendimento pelo Sistema Único de Saúde (SUS) e classe econômica conforme o Critério de Classificação Econômica do Brasil da Associação Brasileira de Empresas de Pesquisa (ABEP). ${ }^{10}$ Tal classificação, que se baseia no acúmulo de bens materiais em casa e na escolaridade do chefe da família, compreende cinco classes econômicas: A (nível mais alto), B, C, D e E (nível mais baixo).

b) História médica - índice de massa corporal (IMC) (calculado por meio de altura e peso autorreferidos); tabagismo (avaliado por meio do relato do paciente ou familiar e definido como ter fumado pelo menos um cigarro no último mês); hipertensão arterial sistêmica, dislipidemia e diabetes (avaliados por meio do relato do paciente ou do familiar mediante diagnóstico médico); e história de infarto prévio, de intervenção coronariana percutânea e de cirurgia de revascularização do miocárdio.

c) Status clínico na admissão hospitalar - sintoma principal (sintoma que motivou o paciente a procurar a emergência do hospital) e seu intervalo (período entre o início do sintoma até a admissão); frequência cardíaca, pressão arterial sistêmica, classificação de Killip, bloqueio atrioventricular total, topografia da isquemia miocárdica e creatinina sérica.

d) Abordagem terapêutica - terapia de reperfusão (fibrinolítico ou intervenção coronariana percutânea), motivos da não reperfusão e medicação adjunta nas primeiras 48 horas.

e) Desfechos clínicos hospitalares - morte, reinfarto, choque cardiogênico, arritmia ventricular, complicações mecânicas, acidente vascular cerebral e sangramento conforme os critérios BARC. ${ }^{11}$

f) Eventos cardiovasculares dentro do primeiro ano após alta hospitalar - morte cardiovascular, infarto agudo do miocárdio ou acidente vascular cerebral.

Com o propósito de identificar os pacientes elegíveis admitidos no hospital de referência do estudo, uma enfermeira especializada em cardiologia e previamente treinada elaborava diariamente uma lista desses pacientes que chegavam ao setor de emergência. Posteriormente, um cardiologista revisava os potenciais casos e os selecionava conforme os critérios de elegibilidade. As características sociodemográficas e a história médica eram registradas pela enfermeira no momento da internação hospitalar ou assim que possível. O status clínico, a abordagem terapêutica e os desfechos clínicos eram avaliados por um cardiologista mediante acompanhamento diário do paciente e revisão do prontuário.

Para a avaliação da ocorrência de eventos cardiovasculares do primeiro ano de seguimento, foi realizado contato telefônico com o paciente 1 ano após a alta hospitalar e, quando apropriado, registros hospitalares foram verificados. Em caso de não respondentes, uma visita em domicílio era realizada. Quando as informações não podiam ser coletadas diretamente com os pacientes, efetuava-se contato com familiar ou pessoa mais próxima.

As informações das hospitalizações eram registradas em formulários impressos e posteriormente digitadas com o uso do programa Microsoft Access. ${ }^{12} \mathrm{O}$ controle de qualidade envolveu revisão desses formulários e checagem de amplitude e de consistência dos dados.

\section{Análise estatística}

Para o cálculo da incidência de hospitalizações por STEMI (casos por 100.000 habitantes por ano), o número de hospitalizações constituiu o numerador e a população do Rio Grande (Censo Demográfico 2010), o denominador. Para as análises ajustadas da incidência de hospitalizações, foi empregada a regressão de Poisson.

Os dados das hospitalizações foram sumarizados em frequência e porcentagem para variáveis categóricas e, no caso para variáveis contínuas, em média/desvio padrão ou mediana/percentis de acordo com a normalidade dos dados (verificação da distribuição por meio do teste de ShapiroWilk). Para a análise ajustada da mortalidade hospitalar, foi utilizado o modelo linear generalizado (família binominal). Para a análise de sobrevida, utilizou-se o método Kaplan-Meier e, para as análises ajustadas, a regressão de Cox. Todas as análises foram ajustadas para medidas repetidas (um paciente com mais de uma hospitalização) ${ }^{13}$ e conduzidas no programa Stata - versão $14.0 .{ }^{14} \mathrm{Um}$ valor de $p$ menor que 0,05 foi considerado para indicar significância estatística.

\section{Resultados}

Durante o período do estudo, ocorreram 575 admissões por sintomas de isquemia miocárdica aguda nas últimas 72 horas associados a SST ao ECG. Dessas, 41 foram excluídas porque os pacientes apresentaram SST transitório enquanto quatro foram descartadas por se tratar de reinfarto. Não houve perdas durante o recrutamento.

\section{Incidência de hospitalizações}

A incidência anual de hospitalização por STEMI no Rio Grande foi de 108 casos por 100.000 habitantes com idade igual ou acima de 25 anos (Tabela 1). A maior taxa foi observada entre homens pertencentes à faixa etária entre 65 e 74 anos. A análise ajustada por sexo mostrou aumento da taxa de hospitalizações conforme aumento da faixa etária ( $p$ para tendência linear $<0,001)$. Em comparação com os pacientes mais jovens, o risco de hospitalização foi 8,9 vezes maior (IC95\% 3,5 - 22,7) no grupo etário de 35 a 44 anos, 28,8 (IC95\% 11,8 - 70,6) no grupo etário de 45 a 54 anos e 64,9 (IC 95\% 26,9 - 156,9) naqueles com 55 anos de idade ou mais.

As incidências anuais de hospitalizações em homens e mulheres foram 159 e 64 casos por 100.000 habitantes, respectivamente. A incidência ajustada por idade foi 2,8 vezes maior em homens que em mulheres (IC95\% 2,3 - 3,3; $\mathrm{p}<0,001)$. 


\begin{tabular}{|c|c|c|c|c|c|c|c|c|}
\hline \multirow[b]{2}{*}{$\begin{array}{l}\text { Idade } \\
\text { (anos) }\end{array}$} & \multicolumn{3}{|c|}{ Homens } & \multicolumn{3}{|c|}{ Mulheres } & \multicolumn{2}{|r|}{ Total } \\
\hline & População & $\begin{array}{l}\text { Número de } \\
\text { Casos }\end{array}$ & $\begin{array}{c}\text { Taxa } \\
\text { (100.000 hab/ano) }\end{array}$ & População & $\begin{array}{l}\text { Número de } \\
\text { Casos }\end{array}$ & $\begin{array}{c}\text { Taxa } \\
\text { (100.000 hab/ano) }\end{array}$ & $\begin{array}{l}\text { Número de } \\
\text { Casos }\end{array}$ & $\begin{array}{c}\text { Taxa } \\
\text { (100.000 hab/ano) }\end{array}$ \\
\hline $25-34$ & 15.609 & 2 & 3 & 16.068 & 3 & 5 & 5 & 4 \\
\hline $35-44$ & 12.550 & 24 & 48 & 13.238 & 12 & 23 & 36 & 35 \\
\hline $45-54$ & 12.485 & 84 & 169 & 14.087 & 34 & 60 & 118 & 111 \\
\hline $55-64$ & 9.486 & 142 & 377 & 10.633 & 53 & 125 & 195 & 243 \\
\hline $65-74$ & 4.601 & 79 & 433 & 6.083 & 27 & 111 & 106 & 249 \\
\hline$\geq 75$ & 2.619 & 33 & 317 & 5.158 & 37 & 180 & 70 & 226 \\
\hline Total & 57.350 & 364 & 159 & 65.267 & 166 & 64 & 530 & 108 \\
\hline
\end{tabular}

\section{Dados das hospitalizações}

Um total de 522 pacientes experimentaram 530 hospitalizações por STEMI - seis pacientes tiveram duas hospitalizações e um paciente teve três hospitalizações. A procura direta pelo hospital foi de $74 \%$ e o atendimento pelo SUS alcançou 85\% das internações.

As características sociodemográficas e a história médica estão demonstradas na Tabela 2. A maioria dos pacientes tinha idade igual ou acima de 55 anos, era do sexo masculino e pertencia à classe econômica C. Quase $50 \%$ dos pacientes eram tabagistas, 59\% tinham hipertensão arterial e 25\% apresentavam diabetes melito.

As características da admissão são descritas na Tabela 3. Cerca de $65 \%$ dos pacientes chegaram dentro de 3 horas do início dos sintomas, enquanto $94 \%$ chegaram dentro de 12 horas. A maioria dos pacientes foi admitida em Killip I e aproximadamente 4\% em Killip IV. O infarto inferior (e inferoposterior) foi responsável por metade dos casos enquanto o anterior extenso representou $16 \%$.

As informações sobre a abordagem terapêutica são apresentadas na Tabela 4. A terapia de reperfusão foi implementada em 80,9\% dos pacientes. Quarenta e quatro pacientes $(8,3 \%)$ não foram considerados elegíveis para terapia de reperfusão, em consequência, na sua maioria, da admissão com mais de 12 horas do início dos sintomas. Entre os pacientes elegíveis, a terapia de reperfusão foi realizada em 88,3\%, e a intervenção coronariana percutânea primária (ICP primária) foi o método preferencial. Não foram submetidos à terapia de reperfusão 11,7\% dos pacientes elegíveis em virtude, principalmente, de motivos desconhecidos. Quase todos os pacientes receberam dupla antiagregação plaquetária e nenhum deles recebeu inibidor da glicoproteína IIb/IIla. Com relação à ICP primária, o uso do acesso radial foi de $69,3 \%$ e o sucesso angiográfico alcançou $94,7 \%$.

Os desfechos clínicos hospitalares estão demonstrados na Tabela 5. Houve 3\% de reinfarto durante a internação hospitalar, quase todos em decorrência de trombose de stent. O choque cardiogênico à admissão e na evolução hospitalar totalizou 9\%. Ocorreram menos de 1\% de complicações mecânicas, de sangramentos e de acidentes vasculares
Tabela 2 - Características sociodemográficas e clínicas dos indivíduos hospitalizados por infarto agudo do miocárdio com supradesnivelamento do segmento de ST $(n=530)$

\begin{tabular}{|c|c|}
\hline Características sociodemográficas & \\
\hline Idade (anos), média (desvio padrão) & $60,4(11,6)$ \\
\hline \multicolumn{2}{|l|}{ Idade (anos), n (\%) } \\
\hline $25-44$ & $41(7,7)$ \\
\hline $45-54$ & $118(22,3)$ \\
\hline $55-64$ & $195(36,8)$ \\
\hline $65-74$ & $106(20,0)$ \\
\hline$\geq 75$ & $70(13,2)$ \\
\hline Sexo masculino, $n(\%)$ & $364(68,7)$ \\
\hline \multicolumn{2}{|l|}{ Nível econômico (ABEP), n (\%) } \\
\hline Classes A e B (mais altos níveis) & $179(33,8)$ \\
\hline Classe C & $268(50,6)$ \\
\hline Classes $\mathrm{D}$ e $\mathrm{E}$ (mais baixos níveis) & $83(15,6)$ \\
\hline \multicolumn{2}{|l|}{ História médica } \\
\hline Sobrepeso, $n(\%)(n=474)$ & $207(43,7)$ \\
\hline Obesidade, $n(\%)(n=474)$ & $112(23,6)$ \\
\hline Tabagismo, n (\%) & $235(44,3)$ \\
\hline Hipertensão arterial sistêmica, $n(\%)(n=500)$ & $295(59,0)$ \\
\hline Diabetes, $n(\%)(n=470)$ & $121(25,7)$ \\
\hline Dislipidemia, $n(\%)(n=456)$ & $203(44,5)$ \\
\hline Infarto do miocárdio prévio, n (\%) & $103(19,9)$ \\
\hline $\begin{array}{l}\text { Revascularização coronariana prévia (cirúrgica e/ou } \\
\text { percutânea), } n(\%)\end{array}$ & $67(12,6)$ \\
\hline
\end{tabular}

ABEP: Associação Brasileira de Empresas de Pesquisa.

cerebrais isquêmicos. Com relação ao tempo de internação hospitalar, observou-se uma mediana de 7 dias (intervalo interquartil de 6 a 10).

A mortalidade hospitalar foi de $8,9 \%$. As taxas de mortalidade conforme grupo etário, sexo e nível socioeconômico, assim como as análises brutas e ajustadas, 


\begin{tabular}{|c|c|}
\hline \multicolumn{2}{|l|}{ Intervalo do sintoma (horas), n (\%) } \\
\hline $0-3$ & $342(64,6)$ \\
\hline$>3-6$ & $79(14,9)$ \\
\hline$>6-12$ & $76(14,3)$ \\
\hline$>12-24$ & $17(3,2)$ \\
\hline$>24-72$ & $16(3,0)$ \\
\hline Frequência cardiaca > 100 bpm, $n(\%)(n=524)$ & $72(13,7)$ \\
\hline PAS $\geq 180 \mathrm{mmHg}$ e/ou PAD $\geq 110 \mathrm{mmHg}, n(\%)(n=516)$ & $114(26,2)$ \\
\hline \multicolumn{2}{|l|}{ Classificação de Killip à admissão, $n(\%)(n=527)$} \\
\hline Killip I & $463(87,8)$ \\
\hline Killip ॥ & $31(5,9)$ \\
\hline Killip III & $10(1,9)$ \\
\hline Killip IV & $23(4,4)$ \\
\hline \multicolumn{2}{|l|}{ Localização do infarto (ECG), n (\%) } \\
\hline Septal, anteroapical e lateral & $140(26,4)$ \\
\hline Anterior extenso & $85(16,0)$ \\
\hline Inferior e inferoposterior & $271(51,2)$ \\
\hline Posterior & $32(6,0)$ \\
\hline Bloqueio de ramo esquerdo novo & $2(0,4)$ \\
\hline Bloqueio AV total, $\mathrm{n}(\%)$ & $21(4,0)$ \\
\hline $\begin{array}{l}\text { Creatinina (mg/dL) à admissão, mediana (intervalo } \\
\text { interquartil) }(n=516)\end{array}$ & $0,97(0,80-1,20)$ \\
\hline
\end{tabular}

estão apresentadas na Tabela 6 . A mortalidade ajustada por sexo e nível econômico foi maior entre os mais velhos ( $p$ para tendência linear $=0,002$ ) alcançando um risco relativo de 3,72 (IC 95\%: 1,57 - 8,82) naqueles com 75 anos ou mais. Embora as estimativas ajustadas indiquem maior mortalidade entre as mulheres (risco relativo 1,21; IC95\%: $0,69-2,14 ; p=0,50)$ e aqueles pertencentes aos mais baixos níveis econômicos (risco relativo 1,66; IC95\%: 0,72 $3,85 ; p=0,24)$, essas diferenças não foram estatisticamente significativas. A mortalidade em 30 dias foi de 9,1\%.

\section{Seguimento clínico}

Informações de 13 entre os 475 pacientes considerados para o seguimento clínico não foram encontradas, o que representa uma perda de seguimento de 2,7\%.

A incidência cumulativa de eventos cardiovasculares no final do primeiro ano de seguimento após alta hospitalar por STEMI foi de $6,1 \%$ (morte cardiovascular, 3,0\%; infarto agudo do miocárdio, 2,4\%; e acidente vascular cerebral, $0,7 \%$ ). A incidência ajustada de eventos cardiovasculares foi maior entre os pacientes com idade igual ou acima de 60 anos (hazard ratio 2,35; IC 95\%: $1,12-4,95 ; p=0,03$ ) (Figura 1 - Painel A). A incidência de eventos cardiovasculares ajustada também foi maior entre as mulheres (hazard ratio 1,55; IC 95\%: 0,77-3,13; $p=0,22$ )
Tabela 4 - Abordagem terapêutica dos indivíduos hospitalizados por infarto agudo do miocárdio com supradesnivelamento do segmento de ST $(n=530)$

\begin{tabular}{lc}
\hline Não elegíveis para terapia de reperfusão, $n(\%)$ & $44(8,3)$ \\
Intervalo do sintoma > 12 horas, $n(\%)$ & $42(95,5)$ \\
Óbito antes da estratégia, $n(\%)$ & $2(0,5)$ \\
Elegíveis submetidos à terapia de reperfusão, $n(\%)$ & $429(88,3)$ \\
ICP primária & $356(83,0)$ \\
ICP primária intencionada, mas não realizada* & $28(6,5)$ \\
Fibrinolítico & $45(10,5)$ \\
Elegíveis não submetidos à terapia de reperfusão, $n(\%)$ & $57(11,7)$ \\
Motivo desconhecido & $50(88,0)$ \\
Reação alérgica ao trombolítico & $6(10,3)$ \\
Sangramento ativo & $1(1,7)$ \\
Acesso transradial na ICP primária, $n(\%)$ & $266(69,3)$ \\
Sucesso angiográfico na ICP primária, $n(\%)$ & $337(94,7)$ \\
Medicação nas primeiras 48 horas, $n(\%)$ & \\
AAS & $523(98,7)$ \\
Clopidogrel & $523(98,7)$ \\
Heparina não fracionada ou heparina de baixo peso & $439(82,8)$ \\
Estatina & $487(91,9)$ \\
Betabloqueador & $411(77,6)$ \\
IECA ou ARA & $379(71,5)$ \\
\hline
\end{tabular}

ICP: intervenção coronariana percutânea; IECA: inibidores da enzima conversora; ARA: antagonistas dos receptores da angiotensina. * Todos pacientes apresentavam fluxo coronariano TIMI 3. Motivos da não realização: opção por cirurgia de revascularização eletiva, opção por ICP eletiva, estenose da lesão-alvo < 50\%, vaso-alvo fino e óbito.

Tabela 5 - Desfecho clínicos hospitalares em indivíduos hospitalizados por infarto agudo do miocárdio com supradesnivelamento do segmento de ST $(n=530)$

\begin{tabular}{lc}
\hline Morte, $\mathrm{n}(\%)$ & $47(8,9)$ \\
Morte cardíaca & $38(7,2)$ \\
Morte não cardíaca (sepse) & $9(1,7)$ \\
Reinfarto, $\mathrm{n}(\%)$ & $16(3,0)$ \\
$\quad$ Trombose de stent, $\mathrm{n}(\%)$ & $15(2,8)$ \\
$\quad$ Trombose pós-uso isolado de extrator de trombo, $\mathrm{n}(\%)$ & $1(0,2)$ \\
Choque cardiogênico (à admissão ou na evolução hospitalar) & $48(9,1)$ \\
Fibrilação ventricular ou taquicardia ventricular (na evolução & $13(2,5)$ \\
hospitalar) & $1(0,2)$ \\
Ruptura de músculo papilar mitral, $\mathrm{n}(\%)$ & $1(0,2)$ \\
Ruptura de septo interventricular, $\mathrm{n}(\%)$ & $1(0,2)$ \\
Ruptura de parede livre, $\mathrm{n}(\%)$ & $5(0,9)$ \\
Sangramento, $\mathrm{n}(\%)$ & $2(0,4)$ \\
$\quad$ BARC Tipo 2 & $3(0,6)$ \\
BARC Tipo 3a & $5(0,9)$ \\
\hline Acidente vascular encefálico isquêmico, $\mathrm{n}(\%)$ &
\end{tabular}




\section{Artigo Original}

\begin{tabular}{|c|c|c|c|c|c|}
\hline & \multirow{2}{*}{ Mortalidade } & \multicolumn{2}{|c|}{ Análise bruta } & \multicolumn{2}{|c|}{ Análise ajustada } \\
\hline & & RR (IC95\%) & Valor $p$ & RR (IC95\%) & Valor $p$ \\
\hline \multicolumn{6}{|l|}{ Idade, anos } \\
\hline $25-54$ & $5,0 \%$ & 1,0 & $<0,001^{*}$ & 1,0 & $0,002^{*}$ \\
\hline $55-64$ & $6,7 \%$ & $1,33(0,56-3,12)$ & & $1,35(0,58-3,16)$ & \\
\hline $65-74$ & $10,4 \%$ & $2,06(0,86-4,97)$ & & $2,01(0,83-4,90)$ & \\
\hline$\geq 75$ & $21,4 \%$ & $4,26(1,89-9,60)$ & & $3,72(1,57-8,82)$ & \\
\hline \multicolumn{6}{|l|}{ Sexo } \\
\hline Masculino & $7,7 \%$ & 1,0 & $0,16 \dagger$ & 1,0 & $0,50^{\dagger}$ \\
\hline Feminino & $11,5 \%$ & $1,49(0,85-2,59)$ & & $1,21(0,69-2,14)$ & \\
\hline \multicolumn{6}{|l|}{ Nível econômico } \\
\hline Classe A e B & $6,2 \%$ & 1,0 & $0,03^{*}$ & 1,0 & $0,24^{*}$ \\
\hline Classe C & $9,0 \%$ & $1,46(0,73-2,90)$ & & $1,34(0,68-2,64)$ & \\
\hline Classe D e E & $14,5 \%$ & $2,35(1,08-5,11)$ & & $1,66(0,72-3,85)$ & \\
\hline
\end{tabular}

RR: risco relativo; IC: intervalo de confiança. * Teste de Wald para tendência linear. ${ }^{\dagger}$ Teste de Wald para heterogeneidade.

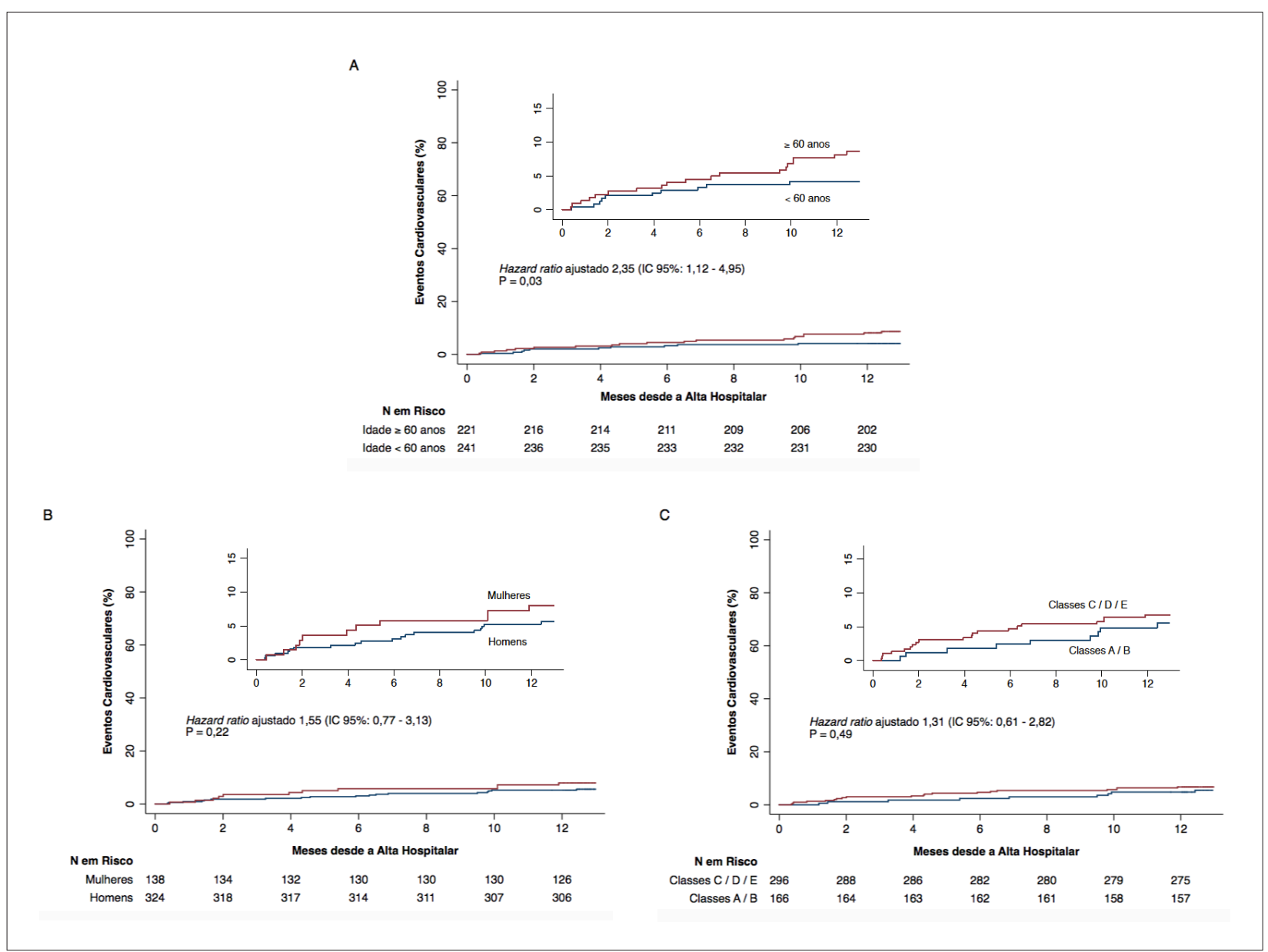

Figura 1 - Incidência acumulativa de eventos cardiovasculares (morte cardiovascular, infarto, acidente vascular cerebral) em 1 ano de seguimento após alta hospitalar por STEMI de acordo com idade (Painel A), sexo (Painel B) e nível econômico (Painel C). 
e entre aqueles pacientes pertencentes aos mais baixos níveis econômicos (hazard ratio 1,31; IC 95\%: 0,61 - 2,82; $p=0,49$ ). No entanto, essas diferenças não atingiram significância estatística (Figura 1 - Painéis B e C). Todas essas estimativas foram ajustadas por idade, sexo, nível econômico e também pela presença de cardiopatia isquêmica prévia, que foi definida, neste estudo, como história de infarto do miocárdio e/ou de revascularização miocárdica (cirúrgica e/ou percutânea). A incidência cumulativa de revascularizações não planejadas (cirúrgicas ou percutâneas) no mesmo período de seguimento foi de $4,7 \%$.

\section{Discussão}

A taxa anual de hospitalização por STEMI foi de 108 casos por 100.000 habitantes; essa taxa foi maior entre os homens com mais de 65 anos de idade. A mortalidade hospitalar e a incidência acumulativa de eventos cardiovasculares em 1 ano foram, respectivamente, 8,9\% e 6,1\%. Ambas as ocorrências foram maiores entre os mais velhos.

A taxa anual de hospitalização por STEMI observada neste estudo foi superior às encontradas em países desenvolvidos. Nos EUA, onde tem havido diminuição dessa incidência ao longo do tempo, ${ }^{3}$ observaram-se taxas iguais a 77 casos por 100.000 habitantes em $2005^{4}$ e 50/100.000 em 2008. ${ }^{3,15}$ $\mathrm{Na}$ Europa, muitos países também apresentaram taxas de hospitalização por STEMI inferiores à encontrada nesse estudo. ${ }^{9}$ Por outro lado, um estudo conduzido em uma cidade da América Latina obteve uma taxa igual a 90 casos por 100.000 habitantes, ${ }^{16}$ próxima à observada no presente estudo. A maior incidência de hospitalização por STEMI em países em desenvolvimento pode decorrer de pior controle dos fatores de risco ${ }^{2}$ e menor acesso e aderência à medicação. ${ }^{17}$ No que diz respeito à maior taxa de hospitalização observada entre os homens e os indivíduos mais velhos, resultados similares também foram encontrados em outros estudos. ${ }^{18,19}$

A taxa de terapia de reperfusão foi próxima às encontradas em países desenvolvidos ${ }^{9,20}$ e superior às observadas em registros nacionais. Esses registros hospitalares que, na maioria, assistem pacientes do sistema público de saúde, mostraram taxas de reperfusão entre 40 e $56 \% .^{21-23}$ Contudo, ainda há uma parcela considerável de pacientes não submetidos à reperfusão, um fato que decorreu principalmente de causas modificáveis. O atraso na busca de assistência médica, bem como o não reconhecimento do quadro clínico de STEMI, são fatores possíveis de melhoria por meio de educação.

No que se refere à mortalidade hospitalar por STEMI, observa-se que varia de acordo com o registro e o país: em registros brasileiros, a mortalidade variou entre 8 e 14\% $\%^{21,22,24,25}$ e, em registros latino-americanos, entre 8 e $11 \% .^{26-30} \mathrm{O}$ mesmo cenário pode ser observado na Europa, onde registros conduzidos em vários países evidenciaram taxas oscilando entre 4 e 13\%. ${ }^{9,31}$ Nos EUA, dois registros mostraram taxas iguais a $5,1 \%{ }^{15}$ e $9,7 \% .^{4}$ Diante desses registros, selecionados de forma não sistemática, a mortalidade por STEMI no presente estudo ficou abaixo dos limites superiores dessas variações. A taxa de reperfusão e o uso da intervenção coronária percutânea por via radial como método preferencial podem ter contribuído para esse resultado.
No entanto, possíveis causas para as variações das taxas de mortalidade entre os estudos devem ser levadas em consideração ao realizar tal análise. As variações das taxas de mortalidade observadas entre os registros podem decorrer do processo metodológico: a) a amostragem de base populacional com registro consecutivo tem menor risco de viés de seleção; b) seleção apenas dos indivíduos que experimentam o primeiro episódio de infarto; c) intervalo do sintoma curto ( $\leq 12$ horas) exclui pacientes com maior risco de morte; d) estudos conduzidos em hospitais com nível terciário de assistência ou em unidades de cuidados intensivos tendem a arrolar pacientes mais graves. Outras causas importantes dessas variações podem ocorrer devido à porcentagem de pacientes submetidos à terapia de reperfusão e ao método dessa terapia utilizado (fibrinolítico ou intervenção coronária percutânea).

A maior mortalidade hospitalar e a maior ocorrência de eventos cardiovasculares em 1 ano de seguimento observada entre os indivíduos mais velhos e do sexo feminino são conhecidas. ${ }^{32,33} \mathrm{~A}$ associação entre esses desfechos e os mais velhos também foi identificada neste estudo. Entretanto, a associação com o sexo feminino não foi estatisticamente significativa - uma justificativa para não detecção pode ser a falta de poder do estudo.

A associação entre nível socioeconômico e desfechos cardiovasculares também é conhecida. ${ }^{34,35}$ Indivíduos com pior condição socioeconômica (baixa escolaridade e baixa renda) tendem a apresentar maior morbimortalidade cardiovascular; essa associação pode ser observada em estudos locais. ${ }^{36-39}$ Do mesmo modo, o presente estudo mostrou maior mortalidade hospitalar e maior incidência de eventos cardiovasculares em um ano entre aqueles pertencentes aos mais baixos níveis socioeconômicos, porém sem significância estatística. Mais uma vez, a falta de poder pode ter influenciado o resultado.

A principal força deste estudo foi a natureza populacional do registro, uma vez que ela permitiu estimar de modo não enviesado a taxa de hospitalização e a mortalidade por STEMI, bem como a ocorrência de eventos cardiovasculares em 1 ano. O recrutamento consecutivo e sem perdas também contribuiu para diminuir a possibilidade de viés de seleção. Outro aspecto relevante que favoreceu as estimativas diretas foi o recrutamento de pacientes com intervalo de até 72 horas, uma vez que indivíduos que se apresentam com maior tempo de dor têm maior risco de morte. Por fim, destaca-se a pequena taxa de perdas na avaliação do seguimento clínico no final do primeiro ano após a alta hospitalar.

Há de se considerar também que este estudo apresenta algumas limitações. Não houve avaliação do intervalo entre o diagnóstico do STEMI e a terapia de reperfusão; esse dado é importante na avaliação da qualidade do atendimento ao paciente com STEMI. Entretanto, dados desse mesmo hospital no período 2005 a 2007 evidenciaram uma mediana de tempo porta-balão igual a 70 minutos (dados não publicados). Outra limitação foi o seguimento clínico obtido por meio de contato telefônico, fato que impediu uma avaliação objetiva dos eventos. No entanto, como o hospital deste estudo é o centro de referência, procurou-se em registros médicos os eventuais eventos cardiovasculares ocorridos. 
Como destacado anteriormente, registros são de fundamental importância. Desse modo, futuros registros devem, com a finalidade de obter estimativas não enviesadas e de comparar com estudos de outros países, ter representatividade da população (seleção randomizada ou inclusão de todos os centros de saúde) e recrutamento consecutivo. ${ }^{5}$ Além disso, recomenda-se selecionar pacientes com um maior tempo de intervalo do sintoma (pelo menos 48 horas).

\section{Conclusão}

Este registro demonstra uma abordagem terapêutica e uma mortalidade hospitalar semelhante às observadas em países desenvolvidos. Entretanto, a taxa de hospitalizações foi maior em comparação a esses países.

\section{Agradecimentos}

Agradecemos à enfermeira Rosa Maria Cacciamani Sousa pelo empenho dedicado à coleta dos dados.

\section{Contribuição dos autores}

Concepção e desenho da pesquisa e Obtenção de dados: Alves L; Análise e interpretação dos dados, Análise

\section{Referências}

1. World Health Organization. Cardiovascular diseases. Geneva:WHO; 2020.

2. Ribeiro AL, Duncan BB, Brant LCC, Lotufo PA, Mill JG, Barreto SM. Cardiovascular health in Brazil: trends and perspectives. Circulation. 2016;133(4):422-33.

3. Yeh RW, Sidney S, Chandra M, Sorel M, Selby JV, Go AS. Population trends in the incidence and outcomes of acute myocardial infarction. N Engl J Med. 2010;362(23):2155-65.

4. McManus DD, Gore J, Yarzebski J, Spencer F, Lessard D, Goldberg RJ. Recent trends in the incidence, treatment, and outcomes of patients with STEMI and NSTEMI. Am J Med. 2011;124(1):40-7.

5. Gitt AK, Bueno H, Danchin N, Fox K, Hochadel M, Kearney P, et al. The role of cardiac registries in evidence-based medicine. Eur Heart J. 2010;31(5):525-9.

6. Writing Group Members, Mozaffarian D, Benjamin EJ, Go AS, ArnettDK, Blaha MJ, et al. Heart disease and stroke statistics-2016 update: a report from the American Heart Association. Circulation. 2016;133(4):e38-360.

7. Bhatt DL, Drozda JP, Jr., Shahian DM, et al. ACC/AHA/STS Statement on the future of registries and the performance measurement enterprise: a report of the American College of Cardiology/American Heart Association Task Force on Performance Measures and The Society of Thoracic Surgeons. J Am Coll Cardiol. 2015;66(20):2230-45.

8. Thygesen K, AlpertJS, Jaffe AS, Simoons ML, Chaitman BR, White HD, etal. Third universal definition of myocardial infarction. Eur HeartJ. 2012;33(20):2551-67.

9. Widimsky P, Wijns W, Fajadet J, Belder M, Knot J, Aaberg L, et al. Reperfusion therapy for ST elevation acute myocardial infarction in Europe: description of the current situation in 30 countries. Eur HeartJ. 2010;31(8):943-57.

10. Associação Brasileira de Empresas de Pesquisa. Critério de Classificação Econômica Brasil. São Paulo; 2010.

11. Mehran R, Rao SV, Bhatt DL, Gibson CM, Caixeta A, Eikelboom J, et al. Standardized bleeding definitions for cardiovascular clinical trials: a consensus report from the Bleeding Academic Research Consortium. Circulation. 2011;123(23):2736-47. estatística, Redação do manuscrito e Revisão crítica do manuscrito quanto ao conteúdo intelectual importante: Alves L, Polanczyk CA

\section{Potencial conflito de interesses}

Declaro não haver conflito de interesses pertinentes.

\section{Fontes de financiamento}

O presente estudo não teve fontes de financiamento externas.

\section{Vinculação acadêmica}

Este artigo é parte de tese de Doutorado de Leonardo Alves pela Universidade Federal do Rio Grande do Sul.

\section{Aprovação ética e consentimento informado}

Este estudo foi aprovado pelo Comitê de Ética da Associação de Caridade Santa Casa de Rio Grande sob o número de protocolo 2.492.526. Todos os procedimentos envolvidos nesse estudo estão de acordo com a Declaração de Helsinki de 1975, atualizada em 2013.
12. Microsoft Access: Release 12.0. Microsoft Corporation; 2007.

13. Kirkwood BR, Sterne JAC. Analysis of clustered data. In: Kirkwood BR, Sterne JAC, eds. Essential Medical Statistics. UK: Blackwell Science; 2003.

14. StataCorp Stata Statistical Software: Release 14.0. Lakeway Drive, College Station TX: Stata Corporation; 2015.

15. Reynolds K, Go AS, Leong TK, Boudreau DM, Cassidy-Bushrow AE, Fortmann SP, et al. Trends in Incidence of hospitalized acute myocardial infarction in the Cardiovascular Research Network (CVRN). Am J Med. 2017;130(3):317-27.

16. Caccavo A, Álvarez A, Bello FH, Ferrari AE, Carrique AM, Lasdica SA, et al. Incidencia poblacional del infarto con elevación del ST o bloqueo de rama izquierda a lo largo de 11 años en una comunidad de la provincia de Buenos Aires. Rev Argent Cardiol. 2007;75(3):185-8.

17. Yusuf S, Islam S, Chow CK, Rangarajan S, Dagenais G, Diaz R, et al. Use of secondary prevention drugs for cardiovascular disease in the community in high-income, middle-income, and low-income countries (the PURE Study): a prospective epidemiological survey. Lancet. 2011;378(9798):1231-43.

18. Roger VL, Weston SA, Gerber Y, Killian JM, Dunlay SM, Jaffe AS, et al. Trends in incidence, severity, and outcome of hospitalized myocardial infarction. Circulation. 2010;121(7):863-9.

19. Fang J, Alderman MH, Keenan NL, Ayala C. Acute myocardial infarction hospitalization in the United States, 1979 to 2005. Am J Med. 2010;123(3):259-66.

20. Roe MT, Messenger JC, Weintraub WS, Cannon CP, Fonarow GC, Dai D, et al. Treatments, trends, and outcomes of acute myocardial infarction and percutaneous coronary intervention. J Am Coll Cardiol. 2010;56(4):254-63.

21. Filgueiras Filho NM, Feitosa Filho GS, Solla DJF, Argôlo FC, Guimarães PO, Paiva Filho IM, et al. Implementation of a regional network for ST-SegmentElevation Myocardial Infarction (STEMI) care and 30-day mortality in a low- to middle-income city in brazil: findings from Salvador's STEMI registry (RESISST). J Am Heart Assoc. 2018;7(14):e008624. 
22. Lana MLL, Beaton AZ, Brant LCC, Bozzi ICRS, Magalhães O, Castro LRA, et al. Factors associated with compliance to AHA/ACC performance measures in a myocardial infarction system of care in Brazil. Int J Qual Health Care. 2017;29(4):499-506.

23. Jose AS, Barreto FH, Oliveira LCS, Oliveira JC, Barreto IDC, Arcelino LAM, et al. Health insurance related disparities in reperfusion and mortality for patients with st segment elevation myocardial infarction in Sergipe, Brazil. J Am Coll Cardiol. 2017;69(11):1267.

24. Marino BCA, Ribeiro ALP, Alkmim MB, Antunes AP, Boersma E, Marcolino MS. Coordinated regional care of myocardial infarction in a rural area in Brazil: Minas Telecardio Project 2. Eur Heart J Qual Care Clin Outcomes. 2016;2(3):215-24

25. Quadros ASd, Schmidt MM, Gazeta CA, Melleu KP, Azmus AD, Teixeira JV et al. Infarto agudo do miocárdio na prática clínica diária. Int J CardiovasC Sci. 2016;29(4):253-61.

26. Nazzal C, Corbalan R, Frenz P, Sepulveda P, Prieto J. Differences in treatment and in-hospital mortality of acute myocardial infarction patients in public and private hospitals in Chile before and after healthcare guarantees. Eur Heart). 2013;34(suppl 1):1026.

27. García Aurelio MJ, Cohen Arazi H, Higa C, Gómez Santa Maria HR, Mauro VM, Fernández H, et al. Infarto agudo de miocardio con supradesnivel persistente del segmento ST: Registro multicéntrico SCAR (Síndromes Coronarios Agudos en Argentina) de la Sociedad Argentina de Cardiología. Rev Argent Cardiol. 2014;82(4):275-84

28. Juan G, Charask A, Perna ER, D’ Imperio H, Bono J, Costa YC, et al. National survey of ST-segment elevation acute myocardial infarction in Argentina (ARGEN-IAM-ST). Rev Argent Cardiol. 2016;84(6):524-33.

29. Martinez-Sanchez C, Borrayo G, Carrillo J, Juarez U, Quintanilla J, Jerjes-Sanches C, et al. Clinical management and hospital outcomes of acute coronary syndrome patients in Mexico: the Third National Registry of Acute Coronary Syndromes (RENASICA III). Arch Cardiol Mex. 2016;86(3):221-32.
30. Chacon-Diaz M, Vega A, Araoz O, Ríos P, Baltodano R, Villanueva F, et al. Epidemiological characteristics of ST-segment elevation myocardial infarction in Peru: Results of the PEruvian Registry of ST-segment Elevation Myocardial Infarction (PERSTEMI). Arch Cardiol Mex. 2018;88(5):403-12.

31. Mandelzweig L, Battler A, Boyko V, Bueno H, Danchin N, Filippatos $\mathrm{G}$, et al. The second Euro Heart Survey on acute coronary syndromes: Characteristics, treatment, and outcome of patients with ACS in Europe and the Mediterranean Basin in 2004. Eur Heart J. 2006;27(19):2285-93.

32. Kyto V, Sipila J, Rautava P. Gender and in-hospital mortality of ST-segment elevation myocardial infarction (from a multihospital nationwide registry study of 31,689 patients). Am J Cardiol. 2015;115(3):303-6.

33. Johansson S, Rosengren A, Young K, Jennings E. Mortality and morbidity trends after the first year in survivors of acute myocardial infarction: a systematic review. BMC Cardiovasc Disord. 2017;17(1):53.

34. Kaplan GA, Keil JE. Socioeconomic factors and cardiovascular disease: a review of the literature. Circulation. 1993;88(4 Pt 1):1973-98.

35. Bhatnagar A. Environmental determinants of cardiovascular disease. Circ Res. 2017;121(2):162-80

36. Bassanesi SL, Azambuja MI, Achutti A. Premature mortality due to cardiovascular disease and social inequalities in Porto Alegre: from evidence to action. Arq Bras Cardiol. 2008;90(6):370-9.

37. Alves L, Cesar JA, Horta BL. Prevalence of angina pectoris in Pelotas, south of Brazil. Arq Bras Cardiol. 2010;95(2):179-85.

38. Ferreira GM, Correia LC, Reis H, Ferreira Filho CB, Freitas F, Ferreira GM, et al. Increased mortality and morbidity due to acute myocardial infarction in a public hospital, in Feira de Santana, Bahia. Arq Bras Cardiol. 2009;93(2):97104.

39. Nazzal C, Corbalán R, Díaz C, Sepúlveda P, Schacht E. Efecto del nivel educacional en la sobrevida posterior a un infarto agudo de miocardio: Registro Chileno de Infarto de Miocardio, GEMI 2009-2012. Rev Méd Chile. 2015;143(7):825-33 